CERN-TH/2002-304

CPT-2002/P.4442

DESY-02-178

IFIC $/ 02-52$

FTUV-02-1111

hep-lat/0211020

\title{
Finite-Size Scaling of Vector and Axial Current Correlators
}

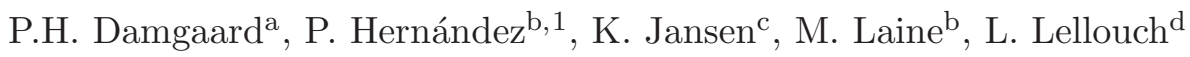 \\ ${ }^{a}$ Niels Bohr Institute, Blegdamsvej 17, DK-2100 Copenhagen Ø, Denmark \\ b Theory Division, CERN, CH-1211 Geneva 23, Switzerland \\ ${ }^{\mathrm{c}}$ NIC/DESY Zeuthen, Platanenallee 6, D-15738 Zeuthen, Germany \\ ${ }^{\mathrm{d}}$ Centre Physique Théorique, CNRS, Case 907, Luminy, F-13288 Marseille, France
}

\begin{abstract}
Using quenched chiral perturbation theory, we compute the long-distance behaviour of twopoint functions of flavour non-singlet axial and vector currents in a finite volume, for small quark masses, and at a fixed gauge-field topology. We also present the corresponding predictions for the unquenched theory at fixed topology. These results can in principle be used to measure the low-energy constants of the chiral Lagrangian, from lattice simulations in volumes much smaller than one pion Compton wavelength. We show that quenching has a dramatic effect on the vector correlator, which is argued to vanish to all orders, while the axial correlator appears to be a robust observable only moderately sensitive to quenching.
\end{abstract}

CERN-TH/2002-304

November 2002

\footnotetext{
${ }^{1}$ On leave from Dept. de Física Teórica, Universidad de Valencia.
} 


\section{Introduction}

The low-energy dynamics of QCD is governed by a spontaneously broken chiral symmetry. Thus, close to the chiral limit, the correlation length associated with the Goldstone bosons is very large. In a finite volume, as required by lattice simulations, this correlation length can easily overtake the linear extent of the box. When this happens, the zero-momentum modes of the Goldstone bosons can no longer be treated perturbatively and finite-size effects become important.

The general procedure for describing this situation in chiral perturbation theory is the $\epsilon$-expansion of Gasser and Leutwyler [1] (see also [2]). It yields precise predictions for the volume and quark-mass dependences of long-distance observables in terms of a few infinite volume low-energy constants. The comparison of these predictions with the volume and mass dependences of the same observables computed in lattice QCD then permits the extraction of the constants. Such lattice studies have become possible recently thanks to the advances with Ginsparg-Wilson formulations [3] of lattice fermions which implement a continuum-like chiral symmetry at finite lattice spacing [4].

In full QCD, all the relevant two-point functions of currents and densities have already been calculated in the $\epsilon$-regime [5, 6]. However, due to the numerical cost of GinspargWilson fermions, lattice simulations in this regime are currently restricted to the quenched approximation. It is therefore important to evaluate the modifications brought about by this approximation. In a previous paper [7, scalar and pseudoscalar correlators were calculated in the quenched approximation, in sectors of fixed topology. We extend that work here by presenting the calculation of flavour non-singlet axial and vector correlators.

At leading order in the chiral expansion, the chiral Lagrangian is parametrized by two lowenergy constants, the chiral condensate $\Sigma$ and the pion decay constant $F$. The value of the first one can be accurately extracted from the spectral density of the low-lying eigenvalues of the Dirac operator (see, e.g., ref. [8]). It has also been obtained by measuring directly the quenched finite-volume quark condensate [9]-[12]. To determine $F$, on the other hand, we note that because it quantifies the strength of the coupling of Goldstone boson fluctuations to the vacuum, it is natural to consider correlation functions of these fluctuations. As shown in [5, 6], the scalar and pseudo-scalar correlation functions are proportional to $\Sigma^{2}$ at leading order, with $F$ appearing at the next-to-leading order. However, in the quenched approximation these correlators also depend on additional unphysical constants $\left(m_{0}^{2}\right.$ and $\left.\alpha\right)$, associated with the flavour singlet field [7], which clearly makes numerical determinations rather difficult, while maybe not impossible [13].

By contrast, the axial and vector current correlators are proportional to $F^{2}$ at leading order, with $\Sigma$ appearing only at the next order. In addition, these correlators are independent of the unknown singlet constants $m_{0}^{2}$ and $\alpha$ at next-to-leading order in the quenched theory. It is therefore expected that these two-point functions will yield particularly clean determinations of (the quenched) $F$. 
Our paper is organized as follows. In Sec. 2 we set up our notation and reiterate a few facts about the $\epsilon$-expansion of chiral perturbation theory. In Secs. 3 and 4 we present our results for flavoured vector and axial current correlators in the quenched theory. Finally, we present in Sec. 5 the analogous results for the full theory, and we conclude in Sec. 6 .

\section{Basic setup}

Let us consider QCD in a toroidal volume $V$ of average length scale $L=V^{1 / 4}$. We assume that the volume is large with respect to the QCD scale, i.e. $F L \gg 1$. As in infinite volume the lightest degrees of freedom are the would-be Goldstone bosons of chiral symmetry breaking. Their interactions can be described in terms of a chiral Lagrangian, which can be systematically expanded in powers of the pion momentum and mass over the cutoff of the effective theory, $\Lambda \simeq 4 \pi F$. To leading order in the chiral expansion,

$$
\mathcal{L}_{\chi P T}=\frac{F^{2}}{4} \operatorname{Tr}\left[\partial_{\mu} U \partial_{\mu} U^{\dagger}\right]-\frac{\Sigma}{2} \operatorname{Tr}\left[U M e^{i \theta / N_{f}}+M^{\dagger} U^{\dagger} e^{-i \theta / N_{f}}\right],
$$

where $U \in \mathrm{SU}\left(N_{f}\right), \theta$ is the vacuum angle, and, to this order, $F$ and $\Sigma$ equal the pseudoscalar decay constant and the chiral condensate, respectively. For simplicity, we consider the quark mass matrix $M$ to be diagonal, $M=\operatorname{diag}(m, \ldots, m)$.

In an infinite volume both the momentum and the pion mass are taken to be of the same order. For a finite $L$ smaller than the inverse of the pion mass, this conventional chiral perturbation theory breaks down when $m \Sigma V \lesssim 1$, because the momentum zero-modes of the pion fields become non-perturbative [1] and require a resummation to all orders. Gasser and Leutwyler provided a procedure to rearrange the chiral expansion appropriately [1]. The first step is to factorise the zero and non-zero modes by writing

$$
U=\exp \left(i \frac{2 \xi}{F}\right) U_{0}
$$

where $U_{0}$ is a constant (in space-time) matrix of $\mathrm{SU}\left(N_{f}\right)$, and the fields $\xi(x)$ parametrize the non-zero-mode manifold. The power counting rules are then those of the so-called $\epsilon$ expansion:

$$
F \sim \mathcal{O}(1), \quad \partial_{\mu} \sim \mathcal{O}(\epsilon), \quad L \sim \mathcal{O}(1 / \epsilon), \quad \xi \sim \mathcal{O}(\epsilon), \quad m \sim \mathcal{O}\left(\epsilon^{4}\right) .
$$

Thus, the quark mass goes like four powers of the momenta, instead of two.

The difficulty of the $\epsilon$-expansion is simply that the integration over $U_{0}$ has to be carried out exactly. Such integrations can however be performed, at least numerically, and results for meson correlators have been computed in full QCD at next-to-leading order in the $\epsilon$ expansion [5, 6]. In some cases, it might be interesting to consider these observables also in sectors of fixed gauge-field topology (particularly when small physical volumes are used). The fixing of topology in chiral perturbation theory amounts to an enlargement of the zero-mode 
manifold from $\mathrm{SU}\left(N_{f}\right)$ to $\mathrm{U}\left(N_{f}\right)$, and to the addition of the term ( $\left.\operatorname{det} U_{0}\right)^{\nu}$ to the weight in the zero-mode integral 14, but then the integration can even be carried out analytically.

Unfortunately, lattice simulations with very light quarks are still restricted to the quenched approximation. Therefore, analytical predictions will also have to be obtained using quenched chiral perturbation theory.

Two recipes have been proposed for dealing with the quenched limit. One is the socalled supersymmetric quenched chiral perturbation theory [15, 16], where the cancellation of internal fermion loops is achieved by adding to the theory ghost bosons of spin $1 / 2$, one for each fermion field. A flavour singlet meson field, $\Phi_{0}$, also needs to be added. The other is the so-called replica method [17, in which only the flavour singlet field $\Phi_{0}$ (whose non-zero momentum modes then correspond to $\operatorname{Tr} \xi$ in Eq. (2.2)) is added, so that the manifold is $\mathrm{U}\left(N_{f}\right)$. Computations are carried out by keeping track separately of the $N_{v}$ external flavours appearing in the operators and of the $N_{f}$ propagating dynamical flavours, and the quenched predictions are obtained by taking the limit $N_{f} \rightarrow 0$, for a fixed $N_{v}$.

While it is believed that the two methods are equivalent in perturbation theory, the situation is somewhat trickier in the $\epsilon$-regime, where a non-perturbative definition of the integration over the zero-mode manifold is required. In particular, the replica method has so far provided results for the zero-mode integrals only in terms of series expansions in a mass parameter (see, however, the recent developments in [18]). Therefore, replica computations are usually transformed into supersymmetric ones at this point.

In the supersymmetric formulation, the zero-mode manifold is obtained by enlarging $\mathrm{U}\left(N_{f}\right)$ to a graded group. The simplest choice is to take $\mathrm{U}\left(N_{f} \mid N_{f}\right)$ with $N_{f}=N_{v}$, although in principle any other choice with a larger $N_{f} \geq N_{v}$ should be equivalent. To leading order in the momentum expansion the supersymmetric quenched chiral Lagrangian is

$$
\mathcal{L}_{Q \chi P T}=\frac{F^{2}}{4} \operatorname{Str}\left[\partial_{\mu} U \partial_{\mu} U^{-1}\right]-\frac{m \Sigma}{2} \operatorname{Str}\left[U_{\theta} U+U^{-1} U_{\theta}^{-1}\right]+\frac{m_{0}^{2}}{2 N_{c}} \Phi_{0}^{2}+\frac{\alpha}{2 N_{c}}\left(\partial_{\mu} \Phi_{0}\right)^{2},
$$

where Str denotes the supertrace, $\Phi_{0} \equiv \frac{F}{2} \operatorname{Str}[-i \ln (U)]$ and $U_{\theta} \equiv \exp \left(i \theta I_{N_{v}} / N_{v}\right)$. Here, $I_{N_{v}}$ is the identity matrix in the fermion-fermion block of "physical" Goldstone bosons and zero otherwise. At this order there are again the two couplings $\Sigma$ and $F$, as in Eq. (2.1), but also a series of new couplings, $m_{0}^{2}, \alpha, \ldots$, associated with the flavour singlet field, which cannot be decoupled in the quenched limit [15, 16.

As discussed in [19, 7], the $\epsilon$-expansion can be set up for this theory as well, at a fixed topological charge $\nu$. The integral over the zero-momentum modes requires, however, special care. To obtain convergent answers, the manifold is in fact not taken to be $\mathrm{U}\left(N_{v} \mid N_{v}\right)$ but what in the mathematics literature is called the maximally symmetric Riemannian submanifold $\widehat{\mathrm{Gl}}\left(N_{v} \mid N_{v}\right)$ [20. The resulting quenched integrals are known explicitly for $N_{v}=1$ [21, 22], and some of them also for $N_{v}=2[23$.

In ref. [7] the scalar and pseudoscalar two-point functions were computed in the $\epsilon$-expansion to next-to-leading order. Below we determine the analogous vector and axial vector corre- 
lators in this expansion, at the same relative order. The reader can find further technical details on the method in [7].

\section{Quenched vector and axial current correlators}

To define the observables considered at the quark level, consider for definiteness a convention where the Euclidean QCD Lagrangian is $\mathcal{L}_{E}=\bar{\psi} \gamma_{\mu} D_{\mu} \psi$, with $\gamma_{\mu}^{\dagger}=\gamma_{\mu},\left\{\gamma_{\mu}, \gamma_{\nu}\right\}=2 \delta_{\mu \nu}$, and $\gamma_{5}=\gamma_{0} \gamma_{1} \gamma_{2} \gamma_{3}$. The vector and axial currents can then be chosen as

$$
V_{\mu}^{a}(x) \equiv i \bar{\psi}(x) T_{N_{v}}^{a} \gamma_{\mu} \psi(x), \quad A_{\mu}^{a}(x) \equiv i \bar{\psi}(x) T_{N_{v}}^{a} \gamma_{\mu} \gamma_{5} \psi(x)
$$

where $T_{N_{v}}^{a}$ are traceless generators in the physical flavour space of the $N_{v}$ valence quarks. We will work with the conventional normalization,

$$
\operatorname{Tr}\left[T_{N_{v}}^{a} T_{N_{v}}^{b}\right]=\frac{1}{2} \delta^{a b} .
$$

The corresponding currents in the chiral theory can be obtained by coupling covariantly the pion field to the external vector $v_{\mu}^{a}$ and axial-vector $a_{\mu}^{a}$ fields, and then taking functional derivatives with respect to those sources. More precisely, the above conventions imply that the partial derivatives in the chiral theory are promoted to covariant ones as

$$
\partial_{\mu} U \rightarrow \partial_{\mu} U+i\left(v_{\mu}^{a}-a_{\mu}^{a}\right) T_{N_{v}}^{a} U-i U T_{N_{v}}^{a}\left(v_{\mu}^{a}+a_{\mu}^{a}\right),
$$

and the currents become

$$
\begin{aligned}
&\left.\left(V_{\mu}^{a}\right)_{\chi \mathrm{PT}} \equiv \mathcal{V}_{\mu}^{a} \equiv\left(\frac{\partial \mathcal{L}_{\chi P T}}{\partial v_{\mu}^{a}}\right)\right|_{v_{\mu}^{a}=0}=-i \frac{F^{2}}{2} \operatorname{Tr}\left[T_{N_{v}}^{a}\left(\partial_{\mu} U U^{-1}+\partial_{\mu} U^{-1} U\right)\right] \\
&\left.\left(A_{\mu}^{a}\right)_{\chi \mathrm{PT}} \equiv \mathcal{A}_{\mu}^{a} \equiv\left(\frac{\partial \mathcal{L}_{\chi P T}}{\partial a_{\mu}^{a}}\right)\right|_{a_{\mu}^{a}=0}=-i \frac{F^{2}}{2} \operatorname{Tr}\left[T_{N_{v}}^{a}\left(-\partial_{\mu} U U^{-1}+\partial_{\mu} U^{-1} U\right)\right] .
\end{aligned}
$$

Inserting here Eq. (2.2), expanding in $\xi$, carrying out the contractions, and taking the replica limit $N_{f} \rightarrow 0$, the two-point functions for $\mathcal{V}_{\mu}^{a}(x), \mathcal{A}_{\mu}^{a}(x)$ are easily determined at

next-to-leading order. The general result can be written in a compact form by introducing $t_{ \pm}^{a} \equiv T_{N_{v}}^{a} \pm U_{0} T_{N_{v}}^{a} U_{0}^{-1}$, and denoting $\mathcal{O}_{\mu}^{a,-}(x) \equiv \mathcal{V}_{\mu}^{a}(x), \mathcal{O}_{\mu}^{a,+}(x) \equiv \mathcal{A}_{\mu}^{a}(x)$, whereby (omitting contact terms)

$$
\begin{aligned}
\left\langle\mathcal{O}_{\mu}^{a, \sigma}(x) \mathcal{O}_{\zeta}^{b, \tau}(0)\right\rangle_{\nu} & =-\frac{F^{2}}{2} \sigma \tau\left\langle\operatorname{Tr}\left[t_{\sigma}^{a} t_{\tau}^{b}\right]\right\rangle_{\nu, U_{0}}^{\mu^{\prime}} \partial_{\mu} \partial_{\zeta} \bar{\Delta}(x-0) \\
-\frac{m \Sigma}{4} \sigma \tau & \left\langle\operatorname{Tr}\left[\left\{t_{\sigma}^{a}, t_{\tau}^{b}\right\}\left(U_{0}+U_{0}^{-1}\right)\right]\right\rangle_{\nu, U_{0}}^{\mu} \int \mathrm{d}^{4} z \partial_{\mu} \bar{\Delta}(z-x) \partial_{\zeta} \bar{\Delta}(z-0) .
\end{aligned}
$$

Here $\langle\cdots\rangle_{\nu, U_{0}}^{\mu}$ denotes the zero-mode average,

$$
\langle\cdots\rangle_{\nu, U_{0}}^{\mu} \equiv \frac{\int_{U_{0}}(\cdots)\left(\operatorname{det} U_{0}\right)^{\nu} \exp \left(\mu \operatorname{Re} \operatorname{Tr}\left[U_{0}\right]\right)}{\int_{U_{0}}\left(\operatorname{det} U_{0}\right)^{\nu} \exp \left(\mu \operatorname{Re} \operatorname{Tr}\left[U_{0}\right]\right)}
$$


while $\bar{\Delta}(x)$ is the meson propagator without zero-momentum modes,

$$
\bar{\Delta}(x) \equiv \frac{1}{V} \sum_{p \neq 0} \frac{e^{i p \cdot x}}{p^{2}} .
$$

Furthermore, $\mu \equiv m \Sigma V$, while $\mu^{\prime}$ is an order- $\epsilon^{2}$ corrected value thereof [7]; in practice, however, $\mu^{\prime}$ is not needed here, since the leading order results turn out to be pure numbers. The first term in Eq. (3.6) is the leading, the second the next-to-leading order contribution. In the supersymmetric formulation the expression is formally identical, after the substitution $\operatorname{Tr} \rightarrow$ Str and the corresponding change of the group manifold for $U_{0}$.

Before we go on to discuss the zero-mode integrations, let us emphasize two important points. First, none of the higher-order couplings of the chiral Lagrangian (the $L_{i}$ 's of Gasser and Leutwyler) contribute at this order. This is in contrast to the standard $p$-expansion of chiral perturbation theory where, for example, $L_{4}$ contributes to the axial correlator at next-to-leading order [5, 6]. This shows the advantage of the $\epsilon$-regime with respect to the $p$ regime in extracting the low energy constants $\Sigma$ and $F$. Second, as shown by a comparison of Eq. (3.6) with the unquenched results (in Sec. 5), quenching does not modify the space-time dependence of these correlators because, remarkably, all potential double-pole contributions to the propagators cancel at both leading and next-to-leading orders. In other words, the additional constants $m_{0}^{2}$ and $\alpha$ of the quenched theory do not appear at all. This is in contrast to the scalar and pseudoscalar correlators, which show more important quenching effects [7]. This implies that the extraction of $\Sigma$ and particularly $F$, which appears at the first order, should be easier from the axial and vector currents.

We now return to the zero-mode integrations in Eq. (3.6). As explained above, the replica method does not yet provide a recipe to compute them, apart from series expansions. However, since the perturbative rules for the two formulations agree, we can at this point switch to the supersymmetric formulation. For a diagonal mass matrix, the results for the zero-mode integrations are invariant in the linear transformation $T_{N_{v}}^{a} \rightarrow g T_{N_{v}}^{a} g^{-1}, T_{N_{v}}^{b} \rightarrow g T_{N_{v}}^{b} g^{-1}$ for any $g \in \widehat{\operatorname{Gl}}\left(N_{v} \mid N_{v}\right)$, and should thus be proportional to the singlet tensor $\delta^{a b}$. For $\left\langle\mathcal{V}_{\mu}^{a}(x) \mathcal{V}_{\zeta}^{b}(0)\right\rangle$ and $\left\langle\mathcal{A}_{\mu}^{a}(x) \mathcal{A}_{\zeta}^{b}(0)\right\rangle$, it is then sufficient to consider

$$
\mathcal{I}_{ \pm}^{0} \equiv\left\langle\operatorname{Str}\left[\left(t_{ \pm}^{a}\right)^{2}\right]\right\rangle_{\nu, U_{0}}^{\mu^{\prime}}, \quad \mathcal{I}_{ \pm}^{1} \equiv\left\langle\operatorname{Str}\left[\left(t_{ \pm}^{a}\right)^{2}\left(U_{0}+U_{0}^{-1}\right)\right]\right\rangle_{\nu, U_{0}}^{\mu},
$$

where the superscript $a$ is not summed over. The superscripts in the $\mathcal{I}$ 's, on the other hand, refer to the order in the expansion at which these contributions enter, as mentioned above.

It is believed that we can now take, without loss of generality, the generators $T_{N_{v}}^{a}$ to be in the simplest group allowing to define flavour non-singlets, i.e. $N_{v}=2$. The first integrals in Eq. (3.9) can then be evaluated by a series of tricks explained in ref. 7]. Using the explicit integrations over $\widehat{\mathrm{Gl}}(2 \mid 2)$ obtained in [23], it is easy to show that for the normalization of Eq. (3.2),

$$
\mathcal{I}_{-}^{0}=0, \quad \mathcal{I}_{+}^{0}=2
$$




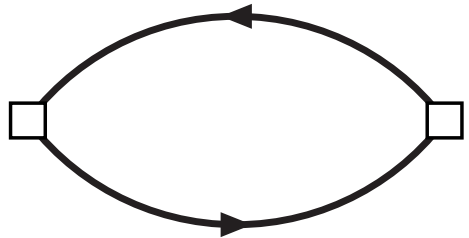

(a)

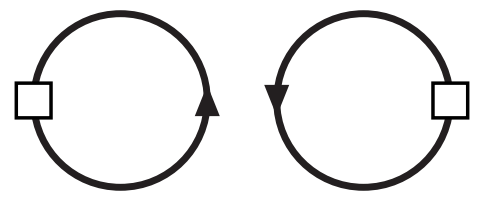

(b)

Figure 1: Two types of external flavour contractions of two quark bilinears: (a) "connected" and (b) "disconnected".

The striking result is that the vector correlator vanishes identically at leading order.

The integrals at the next order are not as easy to obtain, because third derivatives of the partition function for $N_{v}=2$ are needed, and they have to our knowledge not been computed before. We have however performed large and small mass expansions in the replica method using the results of 24, and found that $\mathcal{I}_{-}^{1}$ vanishes to leading order in both of these expansions. (This, in turn, would imply that $\mathcal{I}_{+}^{1}=\left(2 / N_{v}\right)\left\langle\operatorname{Tr}\left[U_{0}+U_{0}^{-1}\right]\right\rangle_{\nu, U_{0}}^{\mu}$.) This can hardly be a coincidence: it suggests that also $\mathcal{I}_{-}^{1}$ is identically zero. It should be noted that in the unquenched theory the non-singlet vector correlator is certainly non-vanishing [5, 6].

In order to understand this feature of the quenched approximation better, we present in the next section a new way of obtaining these results. In fact, we will argue that the vector correlator vanishes in the quenched approximation to all orders in chiral perturbation theory, both in the $p$ - and $\epsilon$-regimes. The same insight provides a useful trick to obtain certain integrals in $\widehat{\mathrm{Gl}}\left(N_{v} \mid N_{v}\right)$ from those based on the much simpler $\widehat{\mathrm{Gl}}(1 \mid 1)$.

\section{Quenched zero-mode integrals and an all-orders argument}

The argument relies on a relation that can be derived at the quark level, where the effect of quenching is immediately obvious. We start by considering the two-point function of any flavour singlet quark bilinear, $\mathcal{O}^{0}(x)$. In general there are two ways of contracting the external flavours leading to two types of diagrams, shown in Fig. 1. In full QCD with $N_{f}$ flavours the first one, which we shall call "connected", will behave in terms of $N_{f}$-counting as $N_{f}+\mathcal{O}\left(N_{f}^{2}\right)$, while the second behaves as $N_{f}^{2}+\mathcal{O}\left(N_{f}^{3}\right)$. The idea is now to isolate the quenched "connected" contribution of one external flavour $\left(N_{v}=1\right)$ by appropriately normalizing the approach of the full singlet quantity to the replica limit $N_{f} \rightarrow 0$,

$$
\left\langle\mathcal{O}^{0}(x) \mathcal{O}^{0}(y)\right\rangle_{\text {connected }}^{\text {quenched, } N_{v}=1}=\lim _{N_{f} \rightarrow 0} \frac{1}{N_{f}}\left\langle\mathcal{O}^{0}(x) \mathcal{O}^{0}(y)\right\rangle^{\text {full }} .
$$

The contribution of the "disconnected" diagram vanishes in this limit since it is $O\left(N_{f}^{2}\right)$.

Now that the quenched connected contribution for one flavour $\left(N_{v}=1\right)$ is isolated, it is straightforward to get any non-singlet $\left(N_{v} \geq 2\right)$ quenched correlator in the limit of degenerate 
quark masses. Indeed, the non-singlet correlators only contain the connected diagram and this diagram gives the same contribution no matter whether the two lines carry the same flavour. In other words, the $N_{v}$ dependence of this diagram is a trivial overall factor, which can easily be provided: the non-singlet quenched correlator is obtained by multiplying Eq. (4.1) with the factor in Eq. (3.2),

$$
\left\langle\mathcal{O}^{a}(x) \mathcal{O}^{b}(y)\right\rangle^{\text {quenched }}=\operatorname{Tr}\left[T_{N_{v}}^{a} T_{N_{v}}^{b}\right] \cdot \lim _{N_{f} \rightarrow 0} \frac{1}{N_{f}}\left\langle\mathcal{O}^{0}(x) \mathcal{O}^{0}(y)\right\rangle^{\text {full }} .
$$

Up to this point we argued at the quark level. However, since the relation of Eq. (4.2) must be valid at any space-time separation, it must hold also in the effective chiral theory. The only subtlety involved in computing the matrix element on the right hand side of Eq. (4.2) is that the chiral Lagrangian for full QCD must include the singlet pseudoscalar field, since the decoupling of the singlet does not commute with the limit $N_{f} \rightarrow 0$.

Assuming that the relation of Eq. (4.2) indeed holds in the effective theory, it is easy to show that the vector correlator vanishes to all orders in the quenched chiral theory ${ }^{2}$. The reason is that, if we were to replace $T_{N_{v}}^{a} \rightarrow T_{N_{f}}^{0} \equiv I_{N_{f}}$ in Eqs. (3.4), (3.5) and write $U=\exp (i 2 \xi / F)$, then $\mathcal{V}_{\mu}^{0}=0$, while $\mathcal{A}_{\mu}^{0}=-2 F \partial_{\mu} \operatorname{Tr} \xi$. In other words, the vector singlet (i.e. baryon number) current vanishes identically ${ }^{3}$, as does then also the limit of Eq. (4.2). On the other hand, the axial singlet current couples to the flavour singlet field, which cannot be decoupled in the quenched limit, leading to a non-vanishing contribution for the axial current.

Since the non-singlet vector current has the quantum numbers of the $\rho$ meson, we note that the result mentioned is consistent with, and in some sense provides justification for, the common lore that the quenched $\rho$ "does not decay".

Let us stress that according to the argument just presented, the zero-mode integrals in the case of degenerate quark masses can all be obtained from the simplest case $N_{v}=1$. In the supersymmetric formulation this implies a set of very non-trivial relations between integrals in $\widehat{\mathrm{Gl}}\left(N_{v} \mid N_{v}\right)$ for various $N_{v}$. In particular, we have checked that one can reproduce in this way all the results for the non-singlet scalar and pseudoscalar correlators of [7], and those in Eqs. (3.10), without the explicit integrals over $\widehat{\mathrm{Gl}}(2 \mid 2)$ of [23]: the only non-trivial integrals needed are in $\widehat{\mathrm{Gl}}(1 \mid 1)$.

Similarly, the quenched singlet correlator for any $N_{v}$ can be easily computed, using the singlet computed for $N_{v}=1$, together with the quenched connected contribution obtained as in Eq. (4.1). The difference of these two quantities is the quenched disconnected contribution. However, once the connected and disconnected contributions for the singlet with $N_{v}=1$ are isolated, the full result for any $N_{v}$ is simply $N_{v} \times($ connected $)+N_{v}^{2} \times($ disconnected $)$. From the point of view of the effective theory, this implies again non-trivial relations between certain integrals in $\widehat{\mathrm{Gl}}\left(N_{v} \mid N_{v}\right)$ and $\widehat{\mathrm{Gl}}(1 \mid 1)$, or the corresponding replica limits of unitary integrals.

\footnotetext{
${ }^{2}$ Obviously there are non-vanishing shorter-distance contributions.

${ }^{3}$ This is true to all orders, not only at the level shown in Eqs. [3.4), (3.5.
} 
For completeness, we illustrate this point in Appendix A.

Using the above observations we can easily compute the integrals $\mathcal{I}_{ \pm}^{1}$ in Eq. (3.9), and thus simplify the expression in Eq. (3.6) $)$. To display the result, it is convenient to consider the zero components of the currents, and to project onto zero spatial momenta by the usual Fourier integration. Using the relations (up to contact terms)

$$
\begin{aligned}
\int_{\mathbf{x}} \partial_{0}^{2} \bar{\Delta}(x-0) & =\frac{1}{T}, \\
\int_{\mathbf{x}} \int_{z} \partial_{0} \bar{\Delta}(z-x) \partial_{0} \bar{\Delta}(z-0) & =T h_{1}\left(\frac{x_{0}}{T}\right),
\end{aligned}
$$

where $T$ is the temporal extent of the box and $h_{1}(\tau) \equiv \frac{1}{2}\left[\left(|\tau|-\frac{1}{2}\right)^{2}-\frac{1}{12}\right]$ [25], we finally obtain (no sum over $a$ ):

$$
\begin{aligned}
C_{v}^{\text {quenched }}\left(x_{0}\right) & \equiv \int_{\mathbf{x}}\left\langle\mathcal{V}_{0}^{a}(x) \mathcal{V}_{0}^{a}(0)\right\rangle_{\nu}=0 \\
C_{a}^{\text {quenched }}\left(x_{0}\right) & \equiv \int_{\mathbf{x}}\left\langle\mathcal{A}_{0}^{a}(x) \mathcal{A}_{0}^{a}(0)\right\rangle_{\nu}=-\frac{F^{2}}{T}\left[1+\frac{2 m \Sigma_{\nu}(\mu) T^{2}}{F^{2}} h_{1}\left(\frac{x_{0}}{T}\right)\right],
\end{aligned}
$$

where the only non-trivial zero-mode integral is the same as appears in the quark condensate obtained with $\widehat{\mathrm{Gl}}(1 \mid 1)[22]$ :

$$
\frac{\Sigma_{\nu}(\mu)}{\Sigma} \equiv \mu\left[I_{\nu}(\mu) K_{\nu}(\mu)+I_{\nu+1}(\mu) K_{\nu-1}(\mu)\right]+\frac{\nu}{\mu}
$$

where $I_{\nu}, K_{\nu}$ are Bessel functions.

To illustrate the results of Eq. (4.6), we show in Fig. 2 the axial correlator normalized to the tree level value, for a realistic lattice setup (of volume $V \equiv T L^{3}$ ). Note that the curvature at zero quark mass limit is quite sizeable, for $\nu>0$.

One apparent paradox in Eqs. (4.5), (4.6) is the following ${ }^{4}$. By performing a global chiral rotation, $\psi \rightarrow \psi^{\prime}=\exp \left(i \phi^{a} T^{a} \gamma_{5}\right) \psi$, which should be a good symmetry in the chiral limit at fixed volume, one can relate the vector and axial correlators through

$$
\sum_{a}\left\langle V_{\mu}^{a}(x) V_{\nu}^{a}(0)\right\rangle=\sum_{a}\left\langle A_{\mu}^{a}(x) A_{\nu}^{a}(0)\right\rangle-\frac{1}{N_{v}} \sum_{a, b, c} f^{a b c}\left\langle V_{\mu}^{a}(x) A_{\nu}^{b}(0) \delta_{c} S\right\rangle,
$$

where $\delta_{c} S=\mathrm{d} S / \mathrm{d} \phi^{c}$ is the variation of the action. The second term on the right is clearly proportional to the bare quark mass, since this is the only source of chiral symmetry breaking, and one might then expect that it vanishes in the chiral limit, leading to equal vector and axial correlators.

\footnotetext{
${ }^{4}$ We thank M. Lüscher for pointing out this paradox.
} 


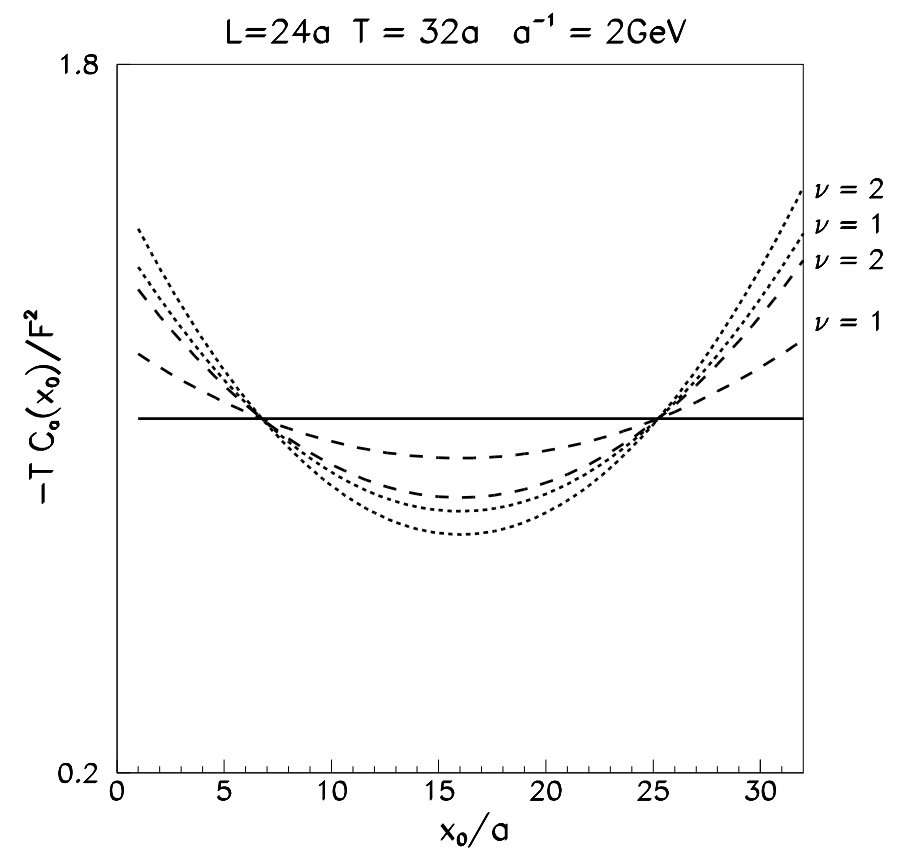

Figure 2: The expression inside the square brackets in Eq. 4.6], for $T=32 a, L=24 a$, $a^{-1}=2 \mathrm{GeV}$, and two masses, $m=0 \mathrm{MeV}$ (dashed) and $m=5 \mathrm{MeV}$ (dotted), as well as a topological charge $\nu=1,2$. The solid line is just the tree-level result. For the parameters in the chiral Lagrangian (which to this order are scale independent) we have assumed $F=93$ $\mathrm{MeV}, \Sigma=(250 \mathrm{MeV})^{3}$.

Clearly, Eqs. (4.5) and (4.6) contradict this expectation. To be explicit we have, in the limit of small quark masses,

$$
\Sigma_{\nu}(\mu) \rightarrow \begin{cases}\Sigma\left(\frac{|\nu|}{\mu}+\frac{\mu}{2|\nu|}\right)+\mathcal{O}\left(\mu^{3}\right), & \nu \neq 0 \\ \Sigma\left(\frac{1}{2}-\gamma+\log (2 / \mu)\right) \mu+\mathcal{O}\left(\mu^{3}\right), & \nu=0\end{cases}
$$

which shows that the axial correlator in Eq. (4.6) does not vanish in this limit. This is however not in contradiction with Eq. (4.8), because the second term on the right does not vanish either. The reason is that when averages are considered in sectors of fixed topology, there are chiral singularities in the three-point function on the right hand side of Eq. (4.8) that cancel the explicit factor of $m$, giving a finite contribution in the chiral limit, which exactly matches the difference between the vector and axial correlators. While the situation for $\nu \neq 0$ is very similar in the full and quenched theories, and these singularities can be clearly identified as the topological zero modes, in the case of $\nu=0$, the behaviours are different in the two cases: the vector and axial correlators are the same in the chiral limit in the full theory while they 
differ in the quenched case. This indicates a more singular chiral behaviour of the quenched theory, which was already noticed in the case of the quark condensate [9, 10.

\section{Results in the full theory}

For completeness and comparison we present in this section the results for the vector and axial correlators in the full theory at fixed topology. They can almost be read from [5], if not because there are a few places where identities were used for the zero-mode integrals that are not valid when averages are taken in sectors of fixed topology. After integrating over space and using the relations

$$
\begin{aligned}
& \mathcal{J}_{-}^{1} \equiv\left\langle\operatorname{Tr}\left[\left(t_{-}^{a}\right)^{2}\left(U_{0}+U_{0}^{\dagger}\right)\right]\right\rangle_{\nu, U_{0}}^{\mu}=\frac{N_{f}}{\mu}\left\langle\operatorname{Tr}\left[\left(t_{+}^{a}\right)^{2}-\left(t_{-}^{a}\right)^{2}\right]\right\rangle_{\nu, U_{0}}^{\mu}, \\
& \mathcal{J}_{+}^{1} \equiv\left\langle\operatorname{Tr}\left[\left(t_{+}^{a}\right)^{2}\left(U_{0}+U_{0}^{\dagger}\right)\right]\right\rangle_{\nu, U_{0}}^{\mu}=\frac{N_{f}}{\mu}\left\langle\operatorname{Tr}\left[\left(t_{-}^{a}\right)^{2}-\left(t_{+}^{a}\right)^{2}\right]\right\rangle_{\nu, U_{0}}^{\mu}+\frac{4}{N_{f}}\left\langle\operatorname{Re} \operatorname{Tr} U_{0}\right\rangle_{\nu, U_{0}}^{\mu},
\end{aligned}
$$

we obtain (no sum over $a$ ):

$$
\begin{aligned}
C_{v}^{\text {full }}\left(x_{0}\right) \equiv \int_{\mathbf{x}}\left\langle\mathcal{V}_{0}^{a}(x) \mathcal{V}_{0}^{a}(0)\right\rangle_{\nu}= & -\frac{F^{2}}{2 T}\left\{\mathcal{J}_{-}^{0}+\frac{N_{f}}{F^{2}}\left(\frac{\beta_{1}}{V^{1 / 2}} \mathcal{J}_{-}^{0}-\frac{T^{2}}{V} k_{00} \mathcal{J}_{+}^{0}\right)\right\}, \\
C_{a}^{\text {full }}\left(x_{0}\right) \equiv \int_{\mathbf{x}}\left\langle\mathcal{A}_{0}^{a}(x) \mathcal{A}_{0}^{a}(0)\right\rangle_{\nu}= & -\frac{F^{2}}{2 T}\left\{\mathcal{J}_{+}^{0}+\frac{N_{f}}{F^{2}}\left(\frac{\beta_{1}}{V^{1 / 2}} \mathcal{J}_{+}^{0}-\frac{T^{2}}{V} k_{00} \mathcal{J}_{-}^{0}\right)\right. \\
+ & \left.\frac{4 \mu}{N_{f} F^{2}} \frac{T^{2}}{V} h_{1}\left(\frac{x_{0}}{T}\right)\left\langle\operatorname{Re} \operatorname{Tr} U_{0}\right\rangle_{\nu, U_{0}}^{\mu}\right\},
\end{aligned}
$$

where $\beta_{1}, k_{00}$ are numerical factors [25], and

$$
\begin{aligned}
\mathcal{J}_{+}^{0} & \equiv\left\langle\operatorname{Tr}\left[\left(t_{+}^{a}\right)^{2}\right]\right\rangle_{\nu, U_{0}}^{\mu^{\prime}}=\frac{1}{N_{f}^{2}-1}\left(N_{f}^{2}-2+\left\langle\operatorname{Tr}\left[U_{0}\right] \operatorname{Tr}\left[U_{0}^{\dagger}\right]\right\rangle_{\nu, U_{0}}^{\mu^{\prime}}\right), \\
\mathcal{J}_{-}^{0} & \equiv\left\langle\operatorname{Tr}\left[\left(t_{-}^{a}\right)^{2}\right]\right\rangle_{\nu, U_{0}}^{\mu^{\prime}}=\frac{1}{N_{f}^{2}-1}\left(N_{f}^{2}-\left\langle\operatorname{Tr}\left[U_{0}\right] \operatorname{Tr}\left[U_{0}^{\dagger}\right]\right\rangle_{\nu, U_{0}}^{\mu^{\prime}}\right) .
\end{aligned}
$$

Here again $\mu=m \Sigma V$, while its order- $\epsilon^{2}$ corrected value is [5, 6]

$$
\mu^{\prime} \equiv \mu \cdot\left(1+\frac{N_{f}^{2}-1}{N_{f}} \frac{\beta_{1}}{F^{2} V^{1 / 2}}\right)
$$

The average left over in Eqs. (5.5), (5.6) is given by [7]

$$
\left\langle\operatorname{Tr}\left[U_{0}\right] \operatorname{Tr}\left[U_{0}^{\dagger}\right]\right\rangle_{\nu, U_{0}}^{\mu^{\prime}}=N_{f}\left[\frac{\Sigma_{\nu}^{\prime}\left(\mu^{\prime}\right)}{\Sigma}+N_{f}\left(\frac{\Sigma_{\nu}\left(\mu^{\prime}\right)}{\Sigma}\right)^{2}+\frac{1}{\mu^{\prime}} \frac{\Sigma_{\nu}\left(\mu^{\prime}\right)}{\Sigma}-N_{f} \frac{\nu^{2}}{\left(\mu^{\prime}\right)^{2}}\right],
$$

where $\Sigma_{\nu}\left(\mu^{\prime}\right)=\left(\Sigma / N_{f}\right)\left\langle\operatorname{Re} \operatorname{Tr} U_{0}\right\rangle_{\nu, U_{0}}^{\mu^{\prime}}$ is the unquenched chiral condensate at fixed topology (see for instance [14]). Note that the unquenched $\Sigma_{\nu}\left(\mu^{\prime}\right) / \Sigma$ depends also on $N_{f}$, although this has not been displayed explicitly. 
As we see from Eq. (5.3), the vector charge is conserved in the full theory just as in the quenched one, but it has now a non-vanishing value. It can be easily checked, however, that at, say, $\nu \neq 0$ but $\mu \rightarrow 0$, one gets $\mathcal{J}_{-}^{0}=0+\mathcal{O}\left(N_{f}^{2}\right), \mathcal{J}_{+}^{0}=2+\mathcal{O}\left(N_{f}^{2}\right), \mathcal{J}_{-}^{1}=0+\mathcal{O}\left(N_{f}\right)$, $\mathcal{J}_{+}^{1}=\left(2 / N_{f}\right)\left\langle\operatorname{Tr}\left[U_{0}+U_{0}^{\dagger}\right]\right\rangle+\mathcal{O}\left(N_{f}\right)$, in accordance with the quenched results in Sec. 4 for $\mathcal{I}_{-}^{0}, \mathcal{I}_{+}^{0}, \mathcal{I}_{-}^{1}, \mathcal{I}_{+}^{1}$, respectively. In the full theory the vector correlator has also been computed at next-to-leading order in the $p$-expansion [5, 6] and, being proportional to $N_{f}$, again vanishes in the replica limit $N_{f} \rightarrow 0$.

\section{Conclusions}

We have presented the results for flavoured vector and axial vector two-point functions in quenched chiral perturbation theory, at next-to-leading order in the $\epsilon$-expansion, corresponding to a finite volume and the vicinity of the chiral limit. Analogous results for unquenched QCD were already presented in [5, 6].

We find that quenching has a striking effect on the vector correlator, which can be argued to vanish to all orders in the chiral expansion. The axial correlator on the other hand depends at the leading order on the low energy constant $F$, and at the next-to-leading order also on $\Sigma$. However, it does not depend to this order on any of the couplings of the quenched theory associated with the singlet pseudoscalar field, $m_{0}^{2}, \alpha, \ldots$, nor on the $L_{i}$ 's of Gasser and Leutwyler. The measurement of the volume and mass dependence of this correlator close to the chiral limit would therefore permit the extraction of (the quenched) $\Sigma$ and $F$, with a minimal contamination from higher order effects.

\section{Acknowledgements}

We warmly thank C. Diamantini, L. Giusti, C. Hoelbling, M. Lüscher, K. Rummukainen, P. Weisz and H. Wittig for useful discussions. This work was supported in part by the European Union Improving Human Potential Programme under contracts No. HPRN-CT2000-00145 ( Hadrons/Lattice QCD ) and HPRN-CT-2002-00311 (EURIDICE).

\section{Appendix A. Examples of quenched zero-mode integrals for $N_{v}>1$}

To illustrate what can be achieved with the method discussed in Sec. 4, we show here how it helps to determine certain integrals with $N_{v}>1$ from known ones with $N_{v}=1$. We do this for the flavour singlet scalar and pseudoscalar correlators, computed for $N_{v}=1$ in [7].

Let us define

$$
S^{0}(x) \equiv \bar{\psi}(x) I_{N_{v}} \psi(x), \quad P^{0}(x) \equiv \bar{\psi}(x) I_{N_{v}} i \gamma_{5} \psi(x)
$$


The correlators of $S^{0}(x), P^{0}(x)$ have two parts: a constant and a space-time dependent contribution. We consider here just the former. As in [7, we denote the constants for $S^{0}, P^{0}$ by $C_{S}^{0}$ and $C_{P}^{0}$, respectively. For $N_{v}=1$ it was found that, to lowest order,

$$
\begin{aligned}
C_{S}^{0} & =\Sigma \Sigma_{\nu}^{\prime}(\mu)+\Sigma^{2}+\frac{\Sigma^{2} \nu^{2}}{\mu^{2}} \\
C_{P}^{0} & =\frac{\Sigma \Sigma_{\nu}(\mu)}{\mu}-\frac{\Sigma^{2} \nu^{2}}{\mu^{2}}
\end{aligned}
$$

where $\Sigma_{\nu}(\mu)$ is the chiral condensate. Using now the prescription of Sec. 4 (Eq. (4.1)), we get the "connected" part of $C_{S}^{0}$ by a $\mathrm{U}\left(N_{f}\right)$ computation,

$$
\begin{aligned}
{\left[C_{S}^{0}\right]_{\text {connected }}^{\text {quenched, } N_{v}=1} } & =\lim _{N_{f} \rightarrow 0} \frac{1}{N_{f}}\left(\frac{\Sigma^{2}}{4}\left\langle\left(\operatorname{Tr}\left[U_{0}+U_{0}^{\dagger}\right]\right)^{2}\right\rangle\right) \\
& =\lim _{N_{f} \rightarrow 0} \frac{1}{N_{f}}\left(\Sigma^{2} N_{f}\left[\frac{\Sigma_{\nu}^{\prime}(\mu)}{\Sigma}+N_{f}\left(\frac{\Sigma_{\nu}(\mu)}{\Sigma}\right)^{2}\right]\right)=\Sigma \Sigma_{\nu}^{\prime}(\mu) .
\end{aligned}
$$

Compared with the $N_{v}=1$ result in Eq. (A.2), this gives the full expression,

$$
\left[C_{S}^{0}\right]^{\text {quenched }}=N_{v} \Sigma \Sigma_{\nu}^{\prime}(\mu)+N_{v}^{2}\left(\Sigma^{2}+\frac{\Sigma^{2} \nu^{2}}{\mu^{2}}\right) .
$$

Similarly, for the constant $C_{P}^{0}$ we get the "connected" part from a $\mathrm{U}\left(N_{f}\right)$ computation,

$$
\begin{aligned}
{\left[C_{P}^{0}\right]_{\text {connected }}^{\text {quenched, } N_{v}=1} } & =-\lim _{N_{f} \rightarrow 0} \frac{1}{N_{f}}\left(\frac{\Sigma^{2}}{4}\left\langle\left(\operatorname{Tr}\left[U_{0}-U_{0}^{\dagger}\right]\right)^{2}\right\rangle\right) \\
& =\lim _{N_{f} \rightarrow 0} \frac{1}{N_{f}}\left(\Sigma^{2} N_{f}\left[\frac{1}{\mu} \frac{\Sigma_{\nu}(\mu)}{\Sigma}-\frac{\nu^{2} N_{f}}{\mu^{2}}\right]\right)=\frac{\Sigma \Sigma_{\nu}(\mu)}{\mu} .
\end{aligned}
$$

Comparing this with the $N_{v}=1$ result, the general expression for any $N_{v}$ is seen to read

$$
\left[C_{P}^{0}\right]^{\text {quenched }}=\frac{N_{v} \Sigma \Sigma_{\nu}(\mu)}{\mu}-\frac{N_{v}^{2} \Sigma^{2} \nu^{2}}{\mu^{2}} .
$$

Eq. (A.7) is in agreement with what one gets from a chiral Ward Identity at fixed topological charge for $N_{v}=2$, as explained in [7]. The space-time dependent parts of the two singlet correlation functions can be constructed for arbitrary $N_{v}$ in a similar way.

\section{References}

[1] J. Gasser and H. Leutwyler, Phys. Lett. B 188 (1987) 477.

[2] H. Neuberger, Phys. Rev. Lett. 60 (1988) 889; Nucl. Phys. B 300 (1988) 180. 
[3] P.H. Ginsparg and K.G. Wilson, Phys. Rev. D 25 (1982) 2649; D.B. Kaplan, Phys. Lett. B 288 (1992) 342 hep-lat/9206013; Y. Shamir, Nucl. Phys. B 406 (1993) 90 hep-lat/9303005; R. Narayanan and H. Neuberger, Nucl. Phys. B 412 (1994) 574 hep-lat/9307006; H. Neuberger, Phys. Lett. B 417 (1998) 141 hep-lat/9707022; ibid. B 427 (1998) 353 hep-lat/9801031; P. Hasenfratz, Nucl. Phys. B ( Proc. Suppl.) 63 (1998) 53 hep-lat/9709110.

[4] M. Lüscher, Phys. Lett. B 428 (1998) 342 hep-lat/9802011.

[5] F.C. Hansen, Nucl. Phys. B 345 (1990) 685.

[6] F.C. Hansen and H. Leutwyler, Nucl. Phys. B 350 (1991) 201.

[7] P.H. Damgaard, C. Diamantini, P. Hernández and K. Jansen, Nucl. Phys. B 629 (2002) 445 hep-lat/0112016.

[8] M.E. Berbenni-Bitsch et al., Phys. Rev. Lett. 80 (1998) 1146 hep-lat/9704018; P.H. Damgaard, U.M. Heller and A. Krasnitz, Phys. Lett. B 445 (1999) 366 hep-lat/9810060; R.G. Edwards et al., Phys. Rev. Lett. 82 (1999) 4188 hep-th/9902117.

[9] P.H. Damgaard et al., Phys. Rev. D 61 (2000) 094503 hep-lat/9907016.

[10] P. Hernández, K. Jansen and L. Lellouch, Phys. Lett. B 469 (1999) 198 hep-lat/9907022 ; P. Hernández et al., JHEP 0107 (2001) 018 hep-lat/0106011.

[11] T. DeGrand [MILC collaboration], Phys. Rev. D 63 (2001) 034503 hep-lat/0007046.

[12] P. Hasenfratz et al., Nucl. Phys. B (Proc. Suppl.) 106 (2002) 751 hep-lat/0109007.

[13] S. Prelovsek and K. Orginos [RBC Collaboration], hep-lat/0209132.

[14] H. Leutwyler and A. Smilga, Phys. Rev. D 46 (1992) 5607.

[15] C.W. Bernard and M.F.L. Golterman, Phys. Rev. D 46 (1992) 853 hep-lat/9204007.

[16] S.R. Sharpe, Phys. Rev. D 46 (1992) 3146 hep-lat/9205020.

[17] P.H. Damgaard and K. Splittorff, Phys. Rev. D 62 (2000) 054509 hep-lat/0003017.

[18] E. Kanzieper, Phys. Rev. Lett. 89 (2002) 250201 cond-mat/0207745;

K. Splittorff and J.J. Verbaarschot, cond-mat/0209594;

Y.V. Fyodorov and G. Akemann, cond-mat/0210647.

[19] P.H. Damgaard, Nucl. Phys. B 608 (2001) 162 hep-lat/0105010.

[20] M.R. Zirnbauer, J. Math. Phys. 37 (1996) 4986 math-ph/9808012. 
[21] J.C. Osborn, D. Toublan and J.J. Verbaarschot, Nucl. Phys. B 540 (1999) 317 hep-th/9806110.

[22] P.H. Damgaard et al., Nucl. Phys. B 547 (1999) 305 hep-th/9811212.

[23] D. Toublan and J.J. Verbaarschot, Nucl. Phys. B 603 (2001) 343 hep-th/0012144.

[24] P.H. Damgaard and K. Splittorff, Nucl. Phys. B 572 (2000) 478 hep-th/9912146;

P.H. Damgaard, Phys. Lett. B 476 (2000) 465 hep-lat/0001002; D. Dalmazi and J.J. Verbaarschot, Nucl. Phys. B 592 (2001) 419 hep-th/0005229.

[25] P. Hasenfratz and H. Leutwyler, Nucl. Phys. B 343 (1990) 241. 